\title{
Retos actuales de la neuroética
}

Current challenges for neuroethics

\author{
JESÚS CONILL SANCHO \\ PEDRO JESÚS PÉREZ ZAFRILLA \\ Universitat De ValËncia
}

El espectacular avance de las neurociencias, gracias al desarrollo de las modernas técnicas de neuroimagen, ha permitido lograr importantes descubrimientos sobre el funcionamiento de nuestro cerebro. De modo especial, se ha ido estableciendo una relación entre la activación de diferentes áreas de la corteza cerebral y nuestros comportamientos en situaciones concretas. Esta conexión ha llevado a numerosos neurocientíficos a creer que el conocimiento de las bases cerebrales del comportamiento humano en los diferentes campos, como la política, la economía, el derecho o la moral, puede explicar cómo funcionan dichos ámbitos. En este sentido han aparecido nuevas disciplinas dedicadas al estudio de las bases cerebrales que hay en cada una de esas esferas. Han surgido así la "neuropolítica», la «neuroeconomía», la «neuroética», el «neuroderecho», y un largo etcétera.

No obstante, esta creciente actividad de los neurocientíficos conlleva numerosas implicaciones de diverso tipo que en la actualidad centran la atención de buena parte de quienes se dedican a la filosofía moral y política. Por un lado, preocupan las consecuencias que estos descubrimientos científicos pueden tener con respecto a nuestra preconcepción como seres libres. Si nuestro comportamiento estuviera determinado por las reacciones neuronales habría que aclarar en qué medida nuestras acciones son producto de decisiones conscientes y libres, y, por tanto, si cabe atribuir algún grado de responsabilidad moral a las personas. Así también, se deberá establecer si, por extensión, los avances neurocientíficos nos permiten conocer cómo funcionan los ámbitos en los que se desenvuelve la acción humana, como la política, la economía o la moral. Otra cuestión que está presente es la relativa, no tanto al conocimiento que de nosotros mismos nos proporcionan las neurociencias, sino a las potencialidades que los nuevos descubrimientos ponen a disposición del ser humano. La aplicación de esos avances científicos dibuja ante nosotros escenarios que mejorarían las actuales condiciones de vida; pero chasta qué punto es aceptable éticamente dicha aplicación? El dopaje genético es un claro ejemplo de ello. Ahora bien, esta pregunta no podrá responderse obviando un tercer 
nivel de discusión: el de los límites que en la actualidad tienen las neurociencias para alcanzar su objetivo central, esto es, explicar tanto el comportamiento humano como el funcionamiento de las distintas esferas sociales, a partir de un método científico experimental propio de las ciencias naturales, aplicado a la activación de la corteza cerebral. Sólo en la medida en que su metodología sea acertada nos dará las claves para reconocer el alcance de esa pretensión central de la neurociencia en sus diversas ramas de aplicación.

Estas cuestiones referidas a la neuroética trazan los ejes centrales del presente número monográfico de la revista Recerca. Revista de Pensament i Anàlisi. Así, el primer artículo, titulado «Speculation and justification in policy-making on neuroenhancement» y realizado Stefan Schlag, examina la evolución del debate surgido en el ámbito de la neuroética sobre el neuroenhancement. El autor fijará tres etapas principales, prestando especial atención a la más reciente. Según Schlag, el debate tiene ahora como principal objeto de atención las consecuencias que tendría el neuroenhancement en un escenario futuro propiciado por el avance de las nuevas tecnologías. Este problema servirá a Schlag para sacar a la luz una cuestión neuroética central: en qué medida es posible justificar las reflexiones éticas asentadas en asunciones meramente especulativas sobre desarrollos futuros de la ciencia. El autor se muestra crítico tanto con la posibilidad de aceptar todo uso de escenarios hipotéticos como con quienes rechazan que sea aceptable ese recurso. Frente a ambas posturas, Schlag mantiene que el debate sigue abierto y que en todo caso el análisis debe hacerse atendiendo a las circunstancias que rodean cada caso particular.

Por otro lado, Arleen Salles en su trabajo «On the normative implications of social neuroscience» cuestiona la relevancia normativa que en realidad tiene la neurociencia. En este sentido, señala que el mismo valor normativo de esta disciplina se ha entendido de un modo ambiguo por parte de los neurocientíficos, llegando a ser interpretado de tres modos distintos. Para la autora ninguna de esas interpretaciones será concluyente, de tal forma que deberá concluirse la insignificancia normativa de la neurociencia en tanto que ésta es un saber descriptivo que no invalida las teorías normativas.

Kathinka Evers y Mariano Sigman abordan en su artículo «Lectura de la mente. Una perspectiva neurofilosófica» la esperanza depositada por la neurociencia en la técnica de lectura de la actividad cerebral para tener un acceso a otras mentes. Los autores reconocen las carencias que a día de hoy poseen las técnicas de neuroimagen para acceder a los contenidos mentales. La complejidad que caracteriza la relación mente-cerebro presenta diversos problemas metodológicos difícilmente abordables para los procedimientos que son empleados en la actualidad. No obstante, Evers y Sigman subrayan la relevancia ética y las potencialidades que adquiriría la posibilidad de inferir estados mentales a partir de los correlatos neuronales, sobre todo por lo 
que hace a la asignación de consciencia a personas con alteraciones de consciencia. En este sentido señalan los autores que los beneficios esperados hacen imperativo seguir investigando en la técnica de la descodificación de la actividad cerebral.

En un sentido similar, Sonia París e Irene Comins en su trabajo «Epistemological and antropological thoughts on neurophilosophy: an initial framework» critican la pretensión de los neurocientíficos de articular las bases biológicas que permitan comprender el comportamiento humano y nuestra forma de realizar juicios morales sobre la realidad. Las autoras señalan los errores metodológicos del proyecto neurocientífico, y las carencias del mismo. La neurociencia mantiene un enfoque reduccionista que desatiende la riqueza de la experiencia y de la naturaleza humana. Ésta es en gran medida maleable y conformada por las relaciones con otros sujetos. Sobre esta base, en la última parte del trabajo las autoras abordan los problemas a los que se enfrenta la pretensión de postular unas bases biológicas universales de la violencia prescindiendo de la diversidad cultural que caracteriza a nuestra especie.

En este carácter reduccionista de la neurociencia incide también Daniel Pallarés con su artículo «Críticas y orientaciones para el estudio de la neuroética». En él presenta diversos aspectos en los que se pone de manifiesto la metodología reduccionista de la neurociencia. Así aborda las limitaciones y el carácter no concluyente de las técnicas que estudian la oxigenación vascular cerebral o la confusión llevada a cabo por los neurocientíficos entre, por un lado, las bases biológicas que predisponen para el comportamiento social y la realización de juicios morales y, por otro, lo que en realidad es el comportamiento moral. Éste, nos recuerda el autor, es un elemento aprendido y desarrollado en sociedad, por lo que no tiene sentido pretender encontrarlo inscrito en nuestra biología.

Por su parte, Edgar Straehle Porras en su artículo «En busca de un fundamento crítico y social de la moral desde una perspectiva neurocientífica» aborda el alcance que los avances producidos en el terreno de la neurociencia tienen para explicar el comportamiento humano. El objetivo principal del autor es rebatir la posición acomodaticia adoptada por aquellos neurocientíficos que creen haber encontrado en las activaciones neuronales de nuestro cerebro la causa real de nuestro comportamiento. Según Porras, estos planteamientos comparten unas mismas tesis reduccionistas. Su respuesta a los mismos partirá de la defensa de una concepción alternativa del cerebro, más rica y abierta a las potencialidades derivadas de nuestra relación con los demás individuos. Es precisamente en la relación interpersonal donde cabe ubicar el mundo moral, por lo que no tendría sentido buscarlo en nuestra biología.

El tema sobre el dopaje genético es objeto del trabajo de Raúl Sebastián y Víctor Páramo «Transhumanistas y bioconservadores en torno al dopaje genético». En él desarrollan la discusión existente entre las dos corrientes principales en torno a este tema, los transhumanistas y los bioconservadores. Los primeros, entre los 
que señalan a Julian Savulescu y Claudio Tamburrini, se muestran favorables a la permisión de estas técnicas de mejoramiento deportivo. Por su parte, los segundos, entre los que incluyen a Michael Sandel y Robert Simon, son claros detractores de las mismas. Terciando en la polémica, Sebastián y Páramo reivindican el valor del espíritu del deporte y la vida del deportista como fin en sí como los límites éticos que cabe poner a la permisión de las prácticas dopantes.

En su artículo «La repugnancia: de la reacción fisiológica a la emoción política» Marta Gil nos expone cómo la repugnancia ha pasado de ser considerada una reacción meramente fisiológica dirigida a facilitar nuestra supervivencia, a adquirir toda una dimensión moral e incluso política. Partiendo de un profundo estudio de autores neurocientíficos como Rozin o McCauley, antropólogos como Frazer y filósofas como Nussbaum, la autora reflexiona sobre el valor cognitivo que posee esta emoción. Gil pondrá un acento especial en el papel que desempeñarán los factores sociales y políticos en la modulación de la repugnancia para ser dirigida no hacia objetos que pueden dañar nuestro organismo, sino hacia individuos o colectivos concretos que se desea señalar como poseedores de un estatus moral inferior. La autora se planteará finalmente en qué medida esta dimensión moral de la repugnancia puede ser educada para dirigirla hacia la condena de las injusticias y no hacia la deshumanización de colectivos concretos.

Por su parte, Jerry Hoeg aporta una lectura diferente de la neuroética en su artículo «Neuroethics and Spanish Literature responses to La crisis». Así como las neurociencias han tratado de explicar los valores, ámbito propio de las humanidades, desde el método científico, Hoeg propone hacer una lectura de la neurociencia desde las humanidades. Más concretamente, presenta la sinergia existente entre los relatos, los mitos y leyendas, por un lado, y nuestro comportamiento, por otro. Así, apoyándose en diversas obras literarias españolas, mantiene el uso adaptativo que el hombre ha hecho de la narrativa desde tiempo inmemorial. Pero también nos recuerda que los efectos que los relatos literarios, políticos o místicos han tenido sobre los hombres son impredecibles, debido a la ambigüedad con que pueden ser interpretados. No hay, por tanto, una interpretación unívoca establecida de antemano. Con ello Hoeg pretenderá criticar el imperialismo realizado por las neurociencias respecto de los demás ámbitos del saber.

El volumen cuenta también con una entrevista realizada por Vicente Ordóñez Roig y Vicent Sanz Rozalén al profesor de la Universidad de Leeds Zygmunt Bauman. En esta entrevista, el pensador de origen polaco nos deja unas interesantes reflexiones sobre la situación en la que se encuentra la democracia representativa en la actualidad. Partiendo de una relación entre los conceptos de política y poder, expone los retos que presenta el fenómeno de la globalización para un modelo de democracia asentado sobre la idea del estado-nación posterior a la paz de Westfalia y que ha llegado a nuestros días. Defenderá que ante una sociedad global en la que el 
poder desborda los límites de los estados no es posible mantener un orden político internacional articulado al servicio de los mismos estados.

La revista incluye finalmente tres reseñas de libros. La primera, realizada por Daniel Pallarés Domínguez, analiza una de las obras que mayor impacto ha adquirido sobre el estudio de la neuroética en lengua española (Neuroética y Neuropolítica. Sugerencias para la educación moral). La segunda reseña, a cargo de Martha M. Rodríguez Coronel, examina una obra profundiza en la relación entre la evolución de las funciones cognitivas de nuestro cerebro y el desarrollo de sistemas morales (Neuroética. Cuando la materia se despierta). Por último, David Muñoz Rodríguez y Emma Gómez Nicolau abordan una sugerente obra sobre la aproximación que realizan nuestros mayores a las nuevas tecnologías (Las generaciones que llegaron tarde. Análisis de las prácticas sociales de los mayores en el ciberespacio). 